\title{
Resolution of disorientation and amnesia during post-traumatic amnesia
}

\author{
Robyn L Tate, Anne Pfaff, Linda Jurjevic
}

\begin{abstract}
Objectives-Despite the growing number of instruments for the prospective measurement of post-traumatic amnesia (PTA) after traumatic brain injury, fundamental issues about the natural history of its resolution and methods of examination remain unresolved. The aims of the present study were to: (1) examine the sequence of resolution of disorientation and amnesia, and (2) determine if the method of measuring the memory component affected the duration of PTA.

Methods-The sample comprised 31 severely injured patients admitted to a brain injury rehabilitation unit who were examined daily until they emerged from PTA. They were administered a composite PTA scale, covering orientation and memory items from standard PTA scales. Patients were consecutively allocated to one of two groups according to the method of measuring the memory component. Each group was administered identical materials with a different procedure.
\end{abstract}

Results-The most common sequence for resolution of disorientation in both groups was person, followed by place, then time. Overall, amnesia resolved before disorientation in $94 \%$ of cases. Correlation coefficients between return of components of orientation and memory were all highly significant, ranging from $r=0.81$ to 0.93 . Significant variability occurred in the number of days to emerge from PTA according to the scale used. There was evidence that the method of measuring memory influenced the patient's capacity to consistently sustain criterion scores on the scale.

Conclusions-These results are contrary to findings in mildly injured patients, in whom orientation usually returns before memory. They also demonstrate that the duration of PTA will be dictated by the method used. These findings raise validity issues with respect to the prospective measurement of PTA, and in particular determining when an individual patient has emerged from PTA, which require further investigation.

(F Neurol Neurosurg Psychiatry 2000;68:178-185)

Keywords: brain injuries; post-traumatic amnesia; confusion; cognition disorders

Levin et $a l^{1}$ described three stages in the recovery process from severe traumatic brain injury, the second stage corresponding to the period of post-traumatic amnesia (PTA), wherein "the patient is confused, amnesic for ongoing events, and likely to evidence behavioural disturbance" (p 675). Determining the duration of PTA is important: it provides an index of the severity of the injury ${ }^{2}$ and is one of the best predictors of the ultimate level of recovery and outcome. ${ }^{3}$ Moreover, many clinical management decisions are made at the time that the patient is considered to have emerged from PTA, such as hospital discharge in less severely injured patients and commencement of formal therapies in the more severely injured.

Despite the clinical and theoretical importance of PTA, there are fundamental issues with respect to the natural history of its resolution and methods of examination which remain unresolved. Most definitions of PTA include reference to the inability to lay down continuous memories, but the methods used to measure this have, with some exceptions, been difficult to operationalise. Other central constructs, such as confusion, are equally difficult to measure reliably, and orientation items seem to be used as a surrogate measure of confusion. This has led some researchers to draw a distinction between the orientation and memory components of PTA and this is reflected in measures of PTA.

Historically, measurement of PTA grew out of clinical judgements. Early approaches ${ }^{45}$ used a retrospective method, based on questioning the patient about his or her memories around the time of the injury. Criticisms have been levelled against this method because of presumed effects of using an unstandardised format (see review by Forrester $e t a l^{6}$ ). Recent investigations, however, have demonstrated that this procedure of evaluating duration of PTA is both valid ${ }^{7}$ and reliable, ${ }^{8}$ particularly for the more severe degrees of injury.

Nonetheless, in rehabilitation settings the prospective evaluation of PTA on a daily basis is usually preferred practice, largely because it provides an ongoing index of the patient's progress. Competing instruments are available, including the Galveston orientation and amnesia test (GOAT), ${ }^{1}$ the scale described by Fortuny and colleagues from Oxford, ${ }^{9}$ the Westmead PTA scale ${ }^{10}$ and its derivatives, ${ }^{112}$ and orientation group monitoring system (OGMS). ${ }^{13}{ }^{14}$ Few comparative studies of these PTA scales have been conducted however, an exception being that by Mysiw et a $l^{15}$ comparing the GOAT and OGMS. Two interrelated issues are often encountered in discussion as to which PTA scale provides the best measurement of PTA: Firstly, post-traumatic disorientation versus post-traumatic amnesia, and sec- 
ondly, the method of measuring the memory component. These issues form the basis of the current paper.

POST-TRAUMATIC DISORIENTATION VERSUS POST-TRAUMATIC AMNESIA

The GOAT has often been criticised because of its focus on orientation items in comparison with items to evaluate anterograde memory. The risk is that patients may be oriented, with scores suggesting that they are out of PTA, yet they may still be acutely amnesic. The seminal study of Gronwall and Wrightson ${ }^{16}$ is usually cited to show that full orientation can occur while patients are still in PTA: of 13 patients who were still amnesic when tested soon after the injury, five $(38 \%)$ were well oriented. This study, however, sampled patients with milder degrees of injury and the generalisation of their findings to more severely injured patients is not known. Moreover, although their study was based on prospective methodology, serially interviewing patients at frequent intervals soon after injury to determine when continuous memory returned, no data were provided in their report to clarify the time interval between return of orientation versus memory. Clearly, the clinical significance attached to these findings will be determined by the latency between return of orientation and memory. If memory returns in close proximity to orientation, albeit lagging slightly, this may be of little consequence.

Another early report comparing the return of orientation and memory is that of Sisler and Penner. ${ }^{17}$ They measured memory using the earliest valid post-trauma memory, an item which has received some criticism because "islands" of memory, which are followed by subsequent deterioration, may be mistaken as signalling the end of PTA. Russell $1^{5}$ notes however, that such islands "are liable to appear when confusion is slight and the patient (is) able to converse and behave in a sensible way" (p 18) . The methodology used by Sisler and Penner may have impacted on their findings, which suggested considerable variability: in $50 \%$ of their sample of 28 cases, disorientation and amnesia resolved simultaneously, while in the remaining patients memory returned before orientation in more cases (29\%) than occurred for orientation returning before memory $(21 \%)$. There have been no reports investigating possible reasons for this variability, and in particular the sequence of return of cognitive functions in PTA has not been reported using the type of anterograde memory items used in the Oxford/Westmead PTA scales.

METHOD OF MEASURING MEMORY DURING PTA Methods of measuring memory in prospective PTA scales differ considerably. Clinical ratings are used in the GOAT and OGMS. In developing their scale, the Westmead group ${ }^{10}$ opted to adapt the Oxford procedure ${ }^{9}$ (using the presentation of three pictures and the examiner's face and name, all to be recalled the following day), in preference to using the GOAT method (recall of the earliest valid post-trauma memory). Like the GOAT, the Westmead scale has the apparent advantage of providing an operational definition of the end of PTA. This has proved to be controversial. One criticism from Wilson et $a l^{18}$ focused on patients who were chronically amnesic (and hence unable to recall the pictures, face, and name the following day) but not confused and disoriented. These patients would be deemed to be in PTA by the Westmead method because they could not achieve the criterion (maximum) score on 3 consecutive days.

Apart from problems in assessing the duration of PTA in those patients who ultimately prove to be chronically amnesic, there are other difficulties with the memory component of the Westmead scale. In the original Oxford procedure, the patient is asked to recall the three pictures presented the previous day. If a perfect score is not obtained using free recall, then a recognition format is administered, wherein the three target pictures are interspersed among five distractor items. There are 21 sets of distractor items, and each day the patient sees the same set of target pictures among new distractors, until the end of the 3rd week, when the cycle is repeated if they are still in PTA. The Westmead procedure differs from the Oxford administration in two important respects. Firstly, in the recognition format there is only a single set of distractor pictures, which are shown to the patient each day. Secondly, when the patient achieves the maximum score (12) on the scale, three new target pictures are selected from the original distractor items, and the old targets become part of the distractor items. The Westmead method (wherein targets are used as foils, and foils as targets), makes the memory component a more demanding task than the Oxford scale wherein, during any 3 week cycle, foils are never used as targets or vice versa. These procedural variations may have implications for determining the end of PTA.

AIMS

Two questions were addressed in the present study: Part 1 of the study investigated the natural history of the resolution of PTA. Specifically, when does amnesia resolve in relation to disorientation? Part 2 of the study was concerned with methodological issues in relation to measuring the memory component of PTA. Specifically, does the method of measuring memory influence the duration of PTA?

\section{Materials and methods}

PARTICIPANTS

Selection criteria for the study comprised the following: Fluency in English, aged between 16 and 65 years at the time of injury, admitted for rehabilitation after a recent traumatic brain injury (TBI), and being in PTA but able to participate in testing. Patients were excluded if there was a history of previous neurological events, psychiatric disorder, or drug/alcohol dependency.

Over a 10 month period in 1997, 40 patients were admitted to the Brain Injury Rehabilitation Unit at Liverpool Hospital in Sydney who 
Table 1 Demographic and injury variables

\begin{tabular}{lll}
\hline & Mean & (SD) \\
\hline Age (y) & 28.42 & $(11.43)$ \\
Initial Glasgow coma scale score & 7.84 & $(3.62)$ \\
& $\mathrm{n}$ & $\%$ \\
Sex: & & \\
$\quad$ Male & 28 & 90.3 \\
$\quad$ Female & 3 & 9.7 \\
Cause of injury: & & \\
$\quad$ RTA & 15 & 48.4 \\
$\quad$ Assault & 7 & 22.6 \\
$\quad$ Fall & 7 & 22.6 \\
$\quad$ Other & 2 & 6.5 \\
Presence of agitated behaviour: & & \\
$\quad$ Yes & 10 & 34.5 \\
$\quad$ No & 19 & 65.5 \\
\hline
\end{tabular}

RTA=Road traffic accident.

met the selection criteria, but nine patients were excluded from the study for the following reasons. Six patients, who were transferred from another hospital, were excluded because they were close to emerging from PTA at the time of admission for rehabilitation. Additionally, the data of three participants were subsequently excluded because in each case it became apparent after months of daily PTA testing that the patients were chronically amnesic, and data collection ceased after the patients were 6 months post-trauma.

Descriptive characteristics of the sample are presented in table 1 . The participants were predominantly male $(90 \%)$ and mostly young, with $58 \%$ less than 30 years of age at the time of injury. The most common cause of injury was road traffic accident (48\%). The severity of injuries varied, with a range in initial Glasgow coma scale (GCS) scores from 3 to 13, but in most cases the injuries were severe, with initial GCS score being 8 or less in $68 \%$. Data from the agitated behaviour scale were available for 29 patients, with the scores of 10 patients above the standard cut off score of 20 , indicative of agitated behaviour. Results of CT were abnormal in 29 cases. The two patients with normal scans had GCS scores of 8 and 13 at admission.

\section{MATERIALS}

Participants were administered a composite PTA scale comprising 24 items covering those in each of the GOAT ${ }^{1}$, Oxford ${ }^{9}$ (F Newcombe, personal communication, 16 September 1991), and Westmead PTA scales (see appendix). The composite scale comprised 15 items covering personal information and orientation, and nine items examining anterograde and retrograde memory. One memory item from the Oxford and Westmead scales was not analysed ("Have you seen my face before?"). It is our clinical experience that it is difficult to be cer- tain of the validity of the response to this item, given that a single clinician usually administers PTA scales and the demand characteristics for patients to answer in the affirmative, irrespective of whether they actually do recall the face. Additionally, documentation of the presence of agitated behaviour was made using the agitated behaviour scale. $^{19}$

In Part 1 of the study, the first aim, investigating the sequence of resolution of disorientation and amnesia, was examined using 12 items from the composite scale. These comprised the seven orientation items used in the study by High et $a l,{ }^{20}$ four items assessing anterograde memory used in the Westmead and Oxford scales, and the GOAT item asking for the first post-trauma memory. The orientation items examined person (name and date of birth), place (city and knowledge that in hospital), and time (correct year, month, and date within 5 days). The anterograde memory items examined recall the following day of the name of the examiner and line drawings of three concrete objects. The memory items were examined using both free recall and recognition formats. Scoring of the orientation items used the procedure of High et al, and orientation was deemed to have returned on the first of 2 consecutive days of perfect (free) recall for each of person, place, and time. For comparison, amnesia was also deemed to have ended on the first of 2 consecutive days of perfect recall of the name of the examiner and three pictures. As suggested by Geffen et $a l,{ }^{21}$ to bypass a scoring artefact in comparing scores for emergence from disorientation versus amnesia, one day was subtracted from each patient's duration of amnesia score, because the second day of testing is the first opportunity to recall the memory items.

The second aim was addressed in part 2 of the study, comparing methods of measuring anterograde memory. Twenty one items from the composite scale were used. The 14 item GOAT was used as the validation measure to compare emergence from PTA with the 12 item Westmead scale, using one of the two administration formats for the three picture items (see Design below). In accordance with standard practice on the Westmead scale, recall of items accepted either free recall or recognition formats. The standard scoring procedures were applied and PTA was deemed to have ended when the patient scored to criterion on the test (the first day of 2 consecutive days scoring above $75 / 100$ on the GOAT and the first of three consecutive days scoring 12/12 on the Westmead).

Table 2 Experimental design

\begin{tabular}{|c|c|}
\hline Group 1 Oxford procedure for memory component using different distractors & Group 2 Westmead procedure for memory component using the same distractors \\
\hline 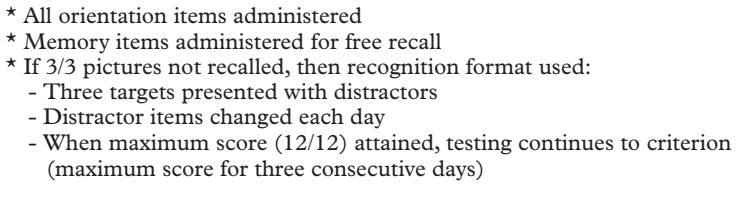 & $\begin{array}{l}\text { * All orientation items administered } \\
\text { * Memory items administered for free recall } \\
\text { * If } 3 / 3 \text { pictures not recalled, then recognition format used: } \\
\text { - Three targets presented with distractors } \\
\text { - Same distractor items used each day } \\
\text { - When maximum score }(12 / 12) \text { attained, new targets are selected from } \\
\text { distractor items, and are replaced by the old targets } \\
\text { - Testing continues to criterion (maximum score for three consecutive days) }\end{array}$ \\
\hline
\end{tabular}


Table 3 Means (SD), with results of t tests between groups 1 and 2, for duration of disorientation, amnesia, and PTA as measured by the GOAT

\begin{tabular}{|c|c|c|c|c|}
\hline & $\begin{array}{l}\text { Group } 1 \text { "different } \\
\text { distractors" }(n=16) \\
M(S D)\end{array}$ & $\begin{array}{l}\text { Group } 2 \text { "same } \\
\text { distractors" }(n=15) \\
M(S D)\end{array}$ & $t$ & $p$ \\
\hline \multicolumn{5}{|c|}{ Day post-trauma on which disorientation resolved: } \\
\hline Person & $14.31(9.58)$ & $16.33(12.06)$ & -0.52 & Ns \\
\hline Place & $18.81(11.18)$ & $21.07(15.29)$ & -0.47 & Ns \\
\hline Time & $23.38(12.76)$ & $26.20(16.97)$ & -0.53 & Ns \\
\hline Full orientation & $23.44(12.65)$ & $27.53(18.23)$ & -0.73 & Ns \\
\hline \multicolumn{5}{|c|}{ Day post-trauma on which amnesia resolved: } \\
\hline Recognition & $19.63(12.09)$ & $19.80(11.97)$ & -0.04 & Ns \\
\hline Free recall & $24.63(18.30)$ & $27.13(21.60)$ & -0.35 & Ns \\
\hline PTA duration with GOAT & $23.94(13.46)$ & $25.67(15.81)$ & -0.33 & Ns \\
\hline
\end{tabular}

PTA $=$ post-traumatic amnesia; GOAT $=$ Galveston orientation and amnesia test

DESIGN

There were two levels of the independent variable group: group 1 participants ("different distractors") learnt the three pictures using the Oxford procedure with the different distractors. Group 2 patients ("same distractors") learnt the three pictures using the Westmead procedure with the same distractors. Table 2 provides a flow diagram of the study design and procedure.

PROCEDURE

Permission to conduct the research was provided by the ethics committee of the South Western Sydney Area Health Service. On admission to the recruiting hospital, the patient's next of kin was approached to provide consent for the patient to be included in the study and consent was obtained for all participants. Patients were consecutively allocated to group 1 or group 2 and were examined each day with the composite PTA scale. It should be noted that the first day of testing did not coincide with the day of the injury for various reasons, such as the patient being in coma, transfer from another hospital, and so forth. On the first day of testing 20 patients were already oriented to person, two were oriented to both person and place, and one to both person and time.

\section{Results}

Initial screening of the data showed that virtually all scores in both groups were normally distributed, for each of demographic, injury, orientation, and memory items. The exceptions occurred in group 1, in which the Kolmogorov-Smirnov Lilliefors statistic was significant for orientation to person $(p<0.04)$, memory for recognition $(\mathrm{p}<0.04)$, and free recall $(\mathrm{p}<0.02)$. Further examination of the skewness and kurtosis of these variables against the normal curve distribution, using the criteria suggested by Tabachnick and Fidell, ${ }^{22}$ indicated that they were not statistically significant.

PART 1: SEQUENCE OF RESOLUTION OF DISORIENTATION AND AMNESIA

For this component of the study, no significant differences between groups 1 and 2 were found for the data from the orientation or memory items, as shown in table 3. Nor were group differences found for demographic variables (age and sex) or injury severity data (initial GCS

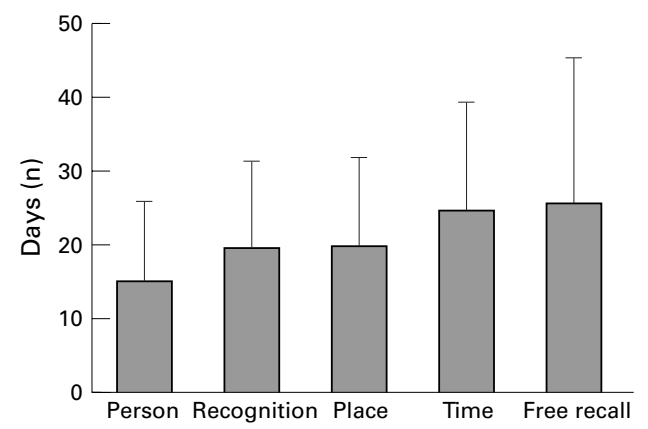

Figure 1 Mean scores (SD) for the day post-trauma on which components of orientation and memory returned.

scores and presence of agitated behaviour). This being the case, the data from the two groups were combined, and are displayed graphically in figure 1. Taking the orientation items first, the combined group data indicated that orientation to person (at day 15 posttrauma), occurred before orientation to place (day 19) and to time (day 24). This order of return of orientation occurred in 16 cases $(51.6 \%)$, with a further six patients being simultaneously oriented to person and place, followed by time, bringing the total to $70.1 \%$. In two of these six cases, the patient was already oriented to person and place on admission to the unit, so that the precise order of return was difficult to determine. A third case was the patient with a normal CT and GCS score of 13 on admission to the unit, with simultaneous orientation to person and place, occurring on day 1 post-trauma. Of the remaining cases, $14 \%$ had as the sequence of resolution, person, then time, followed by place and the final $16 \%$ had other orders, including simultaneous return of the components of orientation.

Results of analyses of both orientation and memory, indicated that, for the group as a whole, recognition memory reached criterion just before return of orientation to place (both at day 19), and five days sooner than orientation to time. Free recall reached criterion the day after orientation to time (day 25 post-trauma). The pattern of resolution of the components of disorientation and amnesia was characterised by marked individual variability. The most common pattern occurred in five cases $(16.1 \%)$, with orientation to person, followed by place, recognition memory, and then orientation to time. A further four cases were simultaneously oriented to person and place, followed by recognition memory, then orientation to time. Recognition memory preceded return of orientation to time in 23 cases $(74.2 \%)$ and orientation to place in 13 cases $(41.9 \%)$. Thus the expected sequence of resolution (disorientation followed by amnesia) was not supported by the data, and in fact occurred in only two people. None the less, association between return of orientation components and memory was strong, all correlation coefficients significant at $\mathrm{p}<0.001$, ranging from $r=0.81$ (orientation to time and free recall memory) to $r=0.93$ (orientation to person and recognition memory). Correlation between return of full orientation and memory was also strong, both for recognition $(r=0.87)$ and free 
Table 4 Means (SD) of the combined sample for duration of disorientation, amnesia, and PTA as measured by the GOAT, along with results of $t$ tests comparing orientation items with recognition and free recall memory

\begin{tabular}{|c|c|c|c|c|c|}
\hline & \multirow{2}{*}{$\begin{array}{l}\text { Combined } \\
\text { groups }(n=31) \\
M(S D)\end{array}$} & \multicolumn{2}{|c|}{ Recognition memory } & \multicolumn{2}{|c|}{ Free recall memory } \\
\hline & & $t$ Value & $p$ Value & $t$ Value & $p$ Value \\
\hline \multicolumn{6}{|l|}{ Orientation: } \\
\hline Person & $15.29(10.72)$ & -5.69 & $<0.001$ & 4.95 & $<0.001$ \\
\hline Place & $19.90(12.15)$ & 0.20 & NS & 3.83 & $<0.002$ \\
\hline Time & $24.74(14.76)$ & -3.66 & $<0.001$ & 0.53 & NS \\
\hline Full orientation & $25.42(15.47)$ & 4.12 & $<0.001$ & 0.23 & NS \\
\hline \multicolumn{6}{|l|}{ Memory: } \\
\hline Recognition & $19.71(11.83)$ & - & - & - & - \\
\hline Free recall & $25.84(19.67)$ & 3.57 & $<0.001$ & - & - \\
\hline PTA duration with GOAT & $24.77(14.42)$ & 4.39 & $<0.001$ & 0.62 & NS \\
\hline
\end{tabular}

PTA=post-traumatic amnesia; GOAT $=$ Galveston orientation and amnesia test

recall $(r=0.85)$. Correlation between recognition memory and free recall was $r=0.94$.

A series of $t$ tests was conducted to determine if the differences in return of components of orientation (person, place, time, and full orientation) versus memory (both recognition and free recall) were statistically significant. Bonferroni adjustments were made to the results for each component of memory (recognition and free recall) separately, to control for an inflated type 1 error rate due to multiple comparisons. The critical $\alpha$ level was set at $\mathrm{p}<0.01(0.05 / 4)$. The results are presented in table 4 , and demonstrate different patterns of significance for recognition versus free recall memory. Taking recognition memory first, there was no significant difference between that and return of orientation to place, however the difference for return of orientation to time was highly significant, as was return of full orientation. In both cases, the mean scores indicate that recognition memory returned before orientation to time and also before full orientation. By contrast, for free recall memory, there was a significant difference between that and return of orientation to place, with the mean scores indicating that orientation to place occurred before free recall. The differences between free recall for both orientation to time, and full orientation were not statistically significant.

Data from the GOAT memory item ("first valid posttrauma memory", first memory) were not analysed in conjunction with the foregoing orientation and memory items because of the many missing data. Almost half the sample $(n=15)$ never attained the criterion on this item before the cessation of PTA testing. It follows that although these patients were out of PTA according to the GOAT, they were still not able to obtain the perfect score on 2 consecutive days for the first memory item. It also follows that, for this subgroup of patients, obtaining criterion on first memory would have occurred after attaining criterion on the orientation items, as well as after attaining criterion on both the recognition and free recall memory items of the Westmead/Oxford type.

The subgroup of subjects who never attained criterion on the first memory item before the cessation of PTA testing had a longer duration of PTA according to the GOAT criterion (day 28 post-trauma) in comparison with the subgroup of subjects who attained criterion on first memory (day 21). The difference, however, was not significant $\left(t_{29}=1.46, \mathrm{p}>0.05\right)$. For the subset of patients who attained criterion on first memory, the pattern of resolution of orientation and memory (recognition and free recall) was very similar to that for the group as a whole, although each of the components occurred earlier post-trauma: person (day 13), recognition memory (day 16), place (day 18), time (day 21), free recall memory (day 21), full orientation (day 22). The first memory item, reaching criterion at day 20, occurred before full orientation and free recall, but after recognition memory. None of the differences, however, were significant. Correlation coefficients between the criterion day for first memory and full orientation $(r=0.57, \quad \mathrm{p}<0.03)$, recognition memory $(r=0.55, \mathrm{p}<0.03)$ and free recall memory $(r=0.67, \mathrm{p}<0.006)$ were statistically significant, but only moderately strong.

PART 2: METHOD OF MEASURING MEMORY

The data of five participants (two from group 1 and three from group 2) who were discharged before scoring to criterion on the Westmead scale (12/12 for 3 consecutive days) were excluded from further analysis. Each of these patients had emerged from PTA according to the GOAT criterion, and was not amnesic in that each patient could correctly recall three of three pictures for 2 consecutive days, as demonstrated in the foregoing section.

Results of an independent samples $t$ test showed that there was no significant difference between the groups on the day to emerge from PTA according to the GOAT, with descriptive data being presented in table 5. Group 2 ("same distractors") took more days to emerge from PTA on the Westmead criterion (day 31) in comparison with group 1 ("different distractors") (day 23), but the difference was not significant. Using paired sample $t$ tests, group 1 showed no statistically significant difference in the number of days to emerge from PTA according to the GOAT or Westmead criteria. By contrast, group 2 took a significantly longer time to emerge from PTA using the Westmead as opposed to the GOAT criteria.

Moreover, an independent samples $t$ test found that group 2 obtained the maximum score on the Westmead scale on significantly more occasions, before the three consecutive scores of 12/12 to indicate the end of PTA, in comparison with group 1 . This result suggests that patients from group 2 were unable to sustain the maximum score for 3 consecutive days. The data of patient 10 , shown in figure 2 , illustrate this point. By day 15 post-trauma he emerged from PTA according to the GOAT, his score always being in excess of 75 . It is noted that he also scored the maximum on the Westmead scale around this time (day 14). However, it was not until a further 2 weeks (day 31 post-trauma) that he attained criterion on the Westmead scale, obtaining the maximum score on seven separate occasions in the interim. 
Table 5 Means (SD), with results of t tests between groups 1 and 2, for variables examining the duration of PTA

\begin{tabular}{|c|c|c|c|c|}
\hline & $\begin{array}{l}\text { Group } 1 \text { "different } \\
\text { distractors" }(n=14) \\
\text { mean }(S D)\end{array}$ & $\begin{array}{l}\text { Group } 2 \text { "same } \\
\text { distractors" }(n=12) \\
\text { mean }(S D)\end{array}$ & $t$ Value & $p$ Value \\
\hline GOAT & $20.79(10.53)$ & $23.83(15.19)$ & -0.60 & NS \\
\hline Westmead scale & $23.86(13.75)$ & $31.33(15.37)$ & -1.31 & NS \\
\hline $\begin{array}{l}\text { Number of occasions maximum Westmead score } \\
\text { obtained before the criterion set }\end{array}$ & $1.14(1.10)$ & $3.33(2.27)$ & -3.05 & $<0.009$ \\
\hline \multicolumn{5}{|l|}{ GOAT $v$ Westmead: } \\
\hline Group 1 difference scores & $-3.07(5.33)$ & - & -2.16 & $\mathrm{p}=0.05$ \\
\hline Group 2 difference scores & - & $-7.50(4.21)$ & -6.17 & $\mathrm{p}<0.001$ \\
\hline
\end{tabular}

PTA=post-traumatic amnesia; GOAT=Galveston orientation and amnesia test.

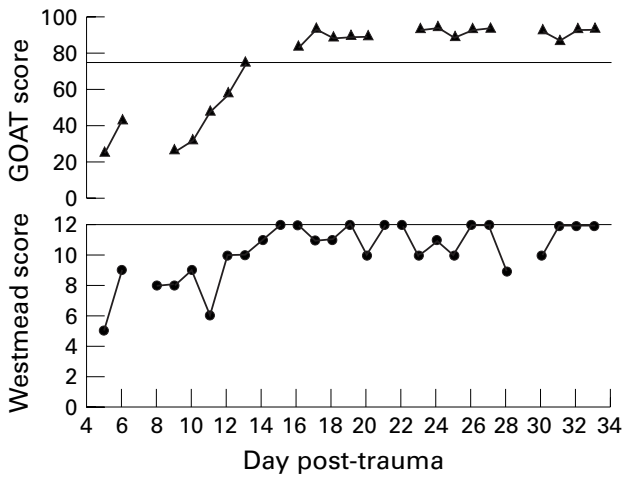

Figure 2 Daily PTA scores of patient 10 (group 2) on the GOAT and Westmead scales.

\section{Discussion}

PART 1: SEQUENCE OF RESOLUTION OF

DISORIENTATION AND AMNESIA

The sequence of return of components of orientation in the present study was consistent with that previously reported by High et $a l^{20}$ who found that $70 \%$ of their patients recovered orientation to person, followed by place, and then time. In the present series, $70 \%$ either had this precise sequence of resolution of components of orientation $(52 \%)$ or were simultaneously oriented to person and place, followed by time $(18 \%)$. Daniel et $a l^{24}$ also found that, in a group of patients receiving electroconvulsive therapy, the sequence of return of components of orientation was significantly different across the three domains, with orientation to person returning before place, followed by time. These investigators, however, also point to an important caveat: that although in general terms orientation to person occurs before time, the specific items show great variability. Hence, of the 12 orientation items used in their study, which were carefully selected to control for specificity, "name" (person) was ranked first, followed by "country" (place) ranked second, then other items examining person; "age" (person) was ranked seventh, after "season" (time) which was ranked sixth. They concluded that this "mixture of order does not support the hypothesis of 'person' returning before 'place', with 'time' returning last" (p178). The separable components within orientation domains recover at different points in time.

Notwithstanding the caveat of Daniel et al, the constancy that was found in the sequence of resolution of the orientation domains did not extend to inclusion of the memory component. The most often occurring pattern of recall (orientation to person, followed by place, then recognition memory, and finally orientation to time) occurred in only $16 \%$ of the sample. Considering return of full orientation, it was rare for orientation to return before (recognition) memory. Hence, the finding of Gronwall and Wrighton ${ }^{16}$ for mildly injured patients, that orientation usually returns before memory, was not supported by these data from severely injured patients. In the present sample, orientation returned before (recognition) memory in only two patients. We do acknowledge, however, that the measure used by Gronwall and Wrightson, return of continuous memories, is different from that on which we base our conclusions, recognition and free recall of new information, and further study of this area is warranted.

A complicating factor emerged from our comparison of the different types of memory items used in PTA scales (first valid posttrauma memory versus recall of newly presented material). We found that half the sample never scored to criterion on the first memory item before the cessation of PTA testing. In this subset of patients, full orientation occurred before criterion would have been attained on this particular memory item, whereas for the remaining half of the group who attained criterion on the first memory item, this occurred before the return of full orientation. These apparently contradictory results with respect to sequence of resolution of amnesia in relation to disorientation underscore the significance of the items selected to measure memory. It is our clinical opinion that, for the purpose of determining whether a patient is amnesic, the recognition format of recalling newly presented information is superior to either of the measures, "first valid post-trauma memory" or "return of continuous memories". Firstly, clinical ratings are not required so that even inexperienced clinicians are able to determine whether or not the patient is laying down new memories. Furthermore, recognition memory is not subject to distortion by other neuropsychological deficits that may be present in the more severely injured patients, such as word finding difficulties or inertia, or physical restraints, such as the presence of tracheostomy tube. Perhaps most importantly, recognition memory format is immediately and easily verifiable.

PART 2: METHOD OF MEASURING MEMORY

The results of the present study also showed the unreliability of determining the end of PTA, which varied according to the PTA scale used, as well as procedural differences in measuring the memory component within a single 
scale. This is demonstrated by the fact that group 2 ("same distractors", as used in the Westmead procedure) obtained the maximum score on the Westmead scale on significantly more occasions than group 1 ("different distractors", as used in the Oxford procedure). Although there were no statistically significant differences with respect to duration of PTA as measured by the Westmead or Oxford procedure for the memory component, there was certainly a trend for group 2 to have a longer duration of PTA using the Westmead items than group 1 (emerging from PTA at days 31 and 23 respectively). The failure for the difference to reach statistical significance may be a function of insufficient power as a consequence of the small sample sizes $(\mathrm{n}=12$ and 14 respectively).

The findings from part 2 of the study raise important validity issues. We suggest there is a subgroup of patients who, like patient 10 in figure 2, "hover" around the maximum score on the Westmead scale. Clinical observation indicates these are the patients who require a recognition format to obtain the correct score on the picture items. They have to distinguish between those pictures they saw the previous day that the examiner wants them to remember, from those pictures that they also saw the previous day (as distractor items) but the examiner does not want them to identify. The difficulty is compounded when the patient obtains the maximum score and then a "new" set of target pictures is presented, but these are already familiar to the patient because they were used as the distractor pictures for the previous targets. Moreover, those pictures that the patient had been previously encouraged to remember now become foils, and they are not to be identified, even though, if a recognition format is required, they will be presented, requiring a temporal distinction. To a patient on the brink of emerging from PTA after a traumatic brain injury, whose memory processes are extremely fragile, and in all likelihood has multiple cognitive deficits, this procedure for examining memory must seem extremely confusing, not to say frustrating.

One of the implications of our results is whether examination using simple memory items of the type used in PTA scales, is really necessary. The inclusion of these items is based on a distinction between "post-traumatic disorientation" and "post-traumatic amnesia". Gronwall and Wrightson ${ }^{17}$ previously questioned the functional significance of posttraumatic disorientation and PTA. Their conclusion was supported by a low and nonsignificant correlation between "degree of disorientation and the duration of PTA" of $r_{\mathrm{s}}=-0.01$, which is in stark contrast to the statistically significant and high correlations in the present study, all in excess of 0.8. Methodological differences between the two studies, however, may have contributed in part to this discrepancy. Gronwall and Wrightson further pointed to the number of dissociations, in which people were well oriented but amnesic and vice versa. As noted earlier in this report, however, in a dynamically resolving condition such as PTA, in which it is expected that as a function of PTA itself both functions will ultimately return to normal levels, it is difficult to operationally define a dissociation. Depending on the lag between resolution of disorientation and amnesia, such a dissociation may not be clinically meaningful. Our results raise the possibility that the distinction between posttraumatic disorientation and PTA may be one of surface labelling and may not make any material difference in determining the end of PTA. Firstly, we found a very close correspondence between the occurrence of full orientation and return of memory, both for recognition memory $(r=0.87)$ and free recall $(r=0.85)$. Moreover, in the vast majority of cases (94\%), the resolution of disorientation occurred after amnesia, using recognition memory format that is standard procedure in the Westmead scale, the difference being statistically significant. Finally, there was no significant difference for the occurrence of full orientation and the stringent criterion of return of free recall memory.

Irrespective of the scale used, the duration of PTA in this sample of patients was in the vicinity of 3 to 4 weeks, indicating that as a group the patients sustained very severe brain injury. It is common knowledge that residual memory disturbance after severe degrees of traumatic brain injury is probably the single most common neuropsychological deficit, Tate $e t a l^{25}$ finding a frequency of $57 \%$ in a consecutive series of severely injured patients examined at 6 years post-trauma. Clearly, when examining the resolution of amnesia during the period of PTA, it is important to distinguish between the type of memory disturbance that occurs in PTA from that which is a residual neuropsychological deficit. In assessing memory in PTA, it is necessary to exercise care that it is not the latter residual memory deficit being assessed. It is useful to be reminded that, in clinical terms, PTA reflects a major disturbance of the sensorium. Jennett and Teasdale ${ }^{26}$ describe the patient who has just emerged from PTA as one who "remembers today what happened yesterday and does not begin each day with a blank mind" (p89). Our data suggest that temporal orientation items, which also involve memory processes in the storage and updating of new information, may provide a more useful index of when a patient emerges from PTA, as distinct from having residual memory deficits. Forrester et $a l^{6}$ make comment about the fact that people without brain damage who are admitted to hospital often do not know the date or day of the week. However, the scoring procedure of GOAT (correct date within 5 days) takes into account such variability, with their scoring criteria being validated against patients admitted to hospital with minor head trauma.

PTA scales definitely have an important place in clinical practice. The scores they yield provide a ready method by which to determine the "depth" of a patient's PTA. Determining the end of PTA, however, seems to be a more complex matter, as previously noted by Glisky and Delaney, ${ }^{27}$ and our results suggest that 
scales in their present form are not completely satisfactory. Further investigation will clarify whether the resolution of PTA can be more simply, reliably, and validly tracked using methods such as orientation markers, as Jackson et $a l^{28}$ have proposed. Certainly, it is much easier to train staff in testing for orientation in comparison with accurate administration and scoring procedures for the memory component of the GOAT, Westmead, or Oxford methods, and places less demands on the acutely confused patient, a large proportion of whom will show agitated behaviour.

This study was funded by a grant from the Motor Accidents Authority of NSW. Thanks are due to Silvia Maggiotto who assisted with data collection, and to Dr Michael Perdices for helpful comments on the paper.

\section{Appendix}

24 items of composite PTA scale. Items comprising each of the three PTA scales are indicated thus: +

\begin{tabular}{llll}
\hline & GOAT & Oxford & Westmead \\
\hline (1) Name & + & - & - \\
(2) Address & + & - & - \\
(3) Age & - & + & + \\
(4) Date of birth & + & + & + \\
(5) Marital status & - & + & - \\
(6) Children & - & + & - \\
(7) Occupation & - & + & - \\
(8) Year & + & + & + \\
(9) Month & + & + & + \\
(10) Day of week & + & + & + \\
(11) Day of month & + & - & - \\
(12) Time of day & + & - & - \\
(13) Period of day & - & - & + \\
(14) Capital city & + & - & - \\
(15) Name of place & + & + & + \\
(16) Admission date & + & - & - \\
(17) Mode of arrival & + & - & - \\
(18) First memory after injury & + & + & - \\
(19) Last memory before injury & + & + & - \\
(20) Recall name & - & + & + \\
(21) Recall face & - & + & + \\
(22-24) Recall 3 pictures & - & + & + \\
\hline
\end{tabular}

PTA=post-traumatic amnesia; GOAT $=$ Galveston orientation and amnesia test

1 Levin HS, O'Donnell VM, Grossman RG. The Galveston orientation and amnesia test. A practical scale to assess cognition after head injury. F Nerv Ment Dis 1979;167:67584.

2 Russell WR, Smith A. Post-traumatic amnesia in closed head injury. Arch Neurol 1961;5:4-17.

3 Bishara SN, Partridge FM, Godfrey HPD, et al. Posttraumatic amnesia and Glasgow coma scale related to out- come in survivors in a consecutive series of patients with closed-head injury Brain Inj 1992;6:373-80.

4 Russell WR. Cerebral involvement in head injury. A study based on the examination of two hundred cases. Brain 1932;55:549-603.

5 Russell WR. The traumatic amnesias. London: Oxford University Press, 1971

6 Forrester G, Encel J, Geffen G. Measuring post-traumatic amnesia (PTA): an historical review. Brain Inj 1994;8:17584.

7 McMillan TM, Jongen ELMM, Greenwood RJ. Assessment of post-traumatic amnesia after severe closed head injury:
retrospective or prospective? $\mathcal{F}$ Neurol Neurosurg Psychiatry 1996;60:422-7.

8 King NS, Crawford S, Wenden FJ, et al. Measurement of post-traumatic amnesia: how reliable is it? 7 Neurol Neurosurg Psychiatry 1997;62:38-42.

9 Artiola i Fortuny L, Briggs M, Newcombe F, et al. Measuring the duration of post traumatic amnesia. $\mathcal{F}$ Neurol Neurosurg Psychiatry 1980;43:377-9.

10 Shores EA, Marosszeky JE, Sandanam J, et al. Preliminary validation of a clinical scale for measuring the duration of validation of a clinical scale for measuring the duration

post-traumatic amnesia. Med F Aust 1986;144:569-72.
11 Horn S, Watson M, Campbell M, et al. Assessing posttrauHorn S, Watson M, Campbell M, et al. Assessing po
matic amnesia. $\mathcal{F}$ Clin Exp Neuropsychol 1991;13:19.

12 Forrester G, Geffen G. Fulia Farr services. Post-traumatic amnesia scales manual. Unley, South Australia: Julia Farr Foundation, 1995.

13 Corrigan JD, Mysiw WJ. Prospective system for monitoring ength of post-traumatic amnesia. Arch Phys Med Rehabil 1984;65:652.

14 Corrigan JD, Arnett JA, Houck LJ, et al. Reality orientation for brain injured patients: group treatment and monitoring of recovery. Arch Phys Med Rehabil 1985;66:675-89.

15 Mysiw WJ, Corrigan JD, Carpenter D, et al. Prospective assessment of posttraumatic amnesia: a comparison of the GOAT and the OGMS. Fournal of Head Trauma Rehabilitation 1990;5:65-72.

16 Gronwall D, Wrightson P. Duration of post-traumatic amnesia after mild head injury. 7 Clin Neuropsychol 1980;2: 51-60.

17 Sisler G, Penner H. Amnesia following severe head injury. Canadian Psychiatric Association fournal 1975;20:333-6.

8 Wilson BA, Baddeley A, Shiel A, et al. How does post-traumatic amnesia differ from the amnesic syndrome and from chronic memory impairment? Neuropsychological Rehabilitation 1992;2:231-43.

19 Corrigan JD. Development of a scale for assessment of agitation following traumatic brain injury. $f$ Clin Exp Neuropsychol 1989;11:261-77.

20 High WM, Levin HS, Gary HE. Recovery of orientation following closed-head injury. F Clin Exp Neuropsychol 1990; 12:703-14.

21 Geffen GM, Encel JS, Forrester GM. Stages of recovery during post-traumatic amnesia and subsequent everyday memory deficits. Neuroreport 1991;2:105-8.

22 Tabachnick BG, Fidell LS. Using multivariate statistics, 3rd ed. New York: HarperCollins, 1996

23 Teasdale G, Jennett B. Assessment of coma and impaired consciousness. Lancet 1974;ii:81-4.

24 Daniel WF, Crovitz HF, Weiner RD. Neuropsychological aspects of disorientation. Cortex 1987;23:169-87.

25 Tate RL, Fenelon B, Manning ML, et al. Patterns of neuropsychological impairment after severe blunt head injury. $\mathcal{F}$ Nerv Ment Dis 1991;179:117-26.

26 Jennett B, Teasdale G. Management of head injuries. Philadelphia: FA Davis, 1981

27 Glisky EL, Delaney SM. Implicit memory and new semantic learning in posttraumatic amnesia. Fournal of Head Trauma Rehabilitation 1996;11:31-42.

28 Jackson WT, Novack TA, Dowler RN. Effective serial measurement of cognitive orientation in rehabilitation: the orientation log. Arch Phys Med Rehabil 1998;78:718-20. 